\title{
Balloon Kyphoplasty: An Effective Treatment for Kummell Disease?
}

\author{
Pius Kim, Seok Won Kim \\ Department of Neurosurgery, Chosun University College of Medicine, Gwangju, Korea
}

\begin{abstract}
Objective: The purpose of this study was to evaluate the efficacy of balloon kyphoplasty for treating Kummel disease accompanying severe osteoporosis.

Methods: Twelve patients with single-level Kummell disease accompanied by severe osteoporosis were enrolled in this investigation. After postural reduction for 1 or 2 days, balloon kyphoplasty was performed on the collapsed vertebrae. Clinical results, radiological parameters, and related complications were assessed at 7 days, 1 month and 6 months after the procedure.

Results: Prior to kyphoplasty, the mean pain score (according to the visual analogue scale) was 8.0. Seven days after the procedure, this score improved to 2.5. Despite the significant improvement compared to preoperative value, the score increased to 4.0 at 6 months after the procedure. The mean preoperative vertebral height loss was $55.4 \%$. Kyphoplasty reduced this loss to $31.6 \%$, but it increased to $38.7 \%$ at 6 months after the procedure. The kyphotic angle improved significantly from $22.4^{\circ} \pm 4.9^{\circ}$ (before the procedure) to $10.1^{\circ} \pm 3.8^{\circ}$ after surgery, However, the improved angle was not maintained 6 months after the procedure. The mean correction loss for the kyphotic deformity was $7.2^{\circ}$ at 6 months after the procedure. Three out of 12 patients sustained adjacent fractures after balloon kyphoplasty within 6 months.

Conclusion: Although balloon kyphoplasty for treating Kummell disease is known to provide stabilization and pain relief, it may be associated with the development of adjacent fractures and aggravated kyphosis.
\end{abstract}

Key Words: Osteonecrosis $\cdot$ Osteoporosis $\cdot$ Kyphoplasty

\section{INTRODUCTION}

The frequency of osteoporotic compression fractures increases with aging. Kummell disease involves vertebral collapse and is a delayed complication of osteoporotic compression fractures. Multiple terms have been used to describe Kummell disease including delayed posttraumatic vertebral osteonecrosis, intravertebral pseudarthrosis, delayed vertebral collapse, and nonunion of a compression fracture, ${ }^{6,712)}$. Regardless of terminology, Kummell disease is not common and most often encountered in patients with severe osteoporosis that have taken longterm courses of corticosteroids or sustained a spinal injury.

Compared to vertebroplasty, balloon kyphoplasty is associated with higher costs but can correct local kyphosis and decrease bone cement extravasation when treating osteoporotic compression fractures. However, the management of Kummell disease accompanying osteoporosis remains controversial and there are no strict guidelines for work-up or treatment. Some

\footnotetext{
- Received: April 1, 2016 • Revised: June 7, 2016

- Accepted: June 14, 2016

Corresponding Author: Seok Won Kim

Department of Neurosurgery, Chosun University College of Medicine,

365 Pilmun-daero, Dong-gu, Gwangju 61453, Korea

Tel: +82-62-220-3126, Fax: +82-62-227-4575

E-mail: ns64902@hanmail.net

(This is an open access article distributed under the terms of the Creative Commons Attribution Non-Commercial License (http://creativecommons.org/licenses/by-nc/4.0/) which permits unrestricted non-commercial use, distribution, and reproduction in any medium, provided the original work is properly cited.
}

authors have reported that excellent pain relief can be expected if the intravertebral cleft (IVC) is filled with bone cement and stabilized because unstable fractures and motion within the IVC produces severe and persistent pain ${ }^{2,3)}$. However, Heo et al. ${ }^{5)}$ insisted that bone cement augmentation for treating Kummell disease is associated with recurrent vertebral collapse as well as dislodged or fragmented bone cement. Moreover, Kummell disease may present with cortical breakage and cord compression, which are contraindicative for cement augmentation ${ }^{11)}$.

So far, few studies have been conducted to prospectively evaluate the clinical outcomes of balloon kyphoplasty for treating Kummell disease in patients with severe osteoporosis. Accordingly, we conducted the current investigation to evaluate the efficacy and safety of balloon kyphoplasty for treating patients with Kummell disease accompanying severe osteoporosis.

\section{MATERIALS AND METHODS}

Twelve patients ( 2 males and 10 females) with Kummell disease accompanying severe osteoporosis were enrolled in this study. Sufficient explanation for the procedure-related complications and approval of an institutional review board of our institute were obtained in each case. The inclusion criteria were (1) neurologically intact individuals despite single-level Kummell disease, (2) patients older than 60 years old, (3) individuals with a severely osteoporotic spine (mean T-score on bone mineral densitometey, <-3.0), and (4) patients with an 
intact posterior vertebral body wall. Kummell disease was diagnosed according to IVC signs on simple lateral radiographs and computed tomography (CT) or evidence of osteonecrosis with a fluid sign on magnetic resonance imaging (MRI). Patients with conditions requiring anterior decompression to ameliorate neurologic deficits, multiple compression fractures, or a history of previous vertebroplasty or spinal surgery were excluded.

\section{Surgical Procedure}

Postural reduction was conducted for 1-2 days using a soft roll under the collapsed vertebra with the patient in the supine position to achieve hyperextension. Approximately $3.0 \mathrm{~mL}$ of polymethylmethacrylate (PMMA) was injected through a kyphoplasty needle (a total of $6.0 \mathrm{~mL}$ per vertebra). The injection was halted if the bone cement caused distention of the entire IVC or leaked into the foraminal vein, disc space, or epidural space. The patients were allowed to move the following day without a back brace. All individuals underwent a physical and

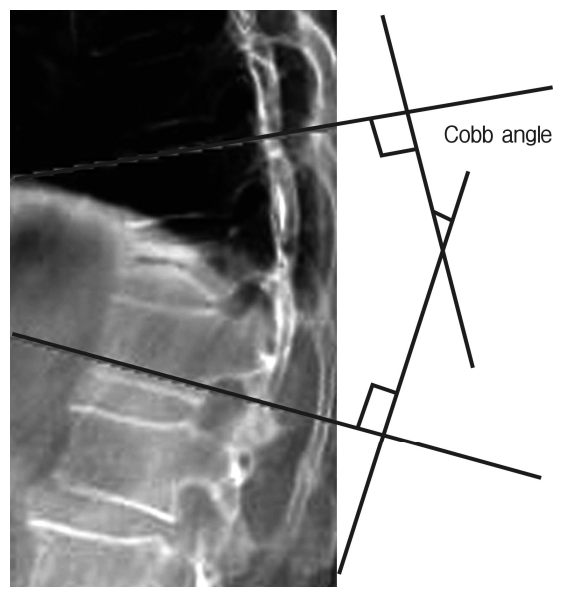

Fig. 1. Measurement of kyphotic angle (Cobb angle). radiological examination prior to surgery, 7 days after the procedure, and 6 months after procedure.

The loss of vertebral height was measured at the site of anterior collapse on lateral radiographs or sagittal CT images. Verteb ral heights are reported as fractions relative to the reference height of adjacent lower vertebra. Kyphotic angle (Cobb angle) was measured by drawing a straight line from the superior endplate one level above the fractured body and a straight line from the inferior endplate one level below the fractured body (Fig. 1). The angle was measured in all except one patient who was not diagnosed with kyphosis because the L3 vertebra was involved. Imaging and clinical findings including the vertebral level, vertebral height restoration, evidence of local kyphosis, clinical outcomes, and complications were analyzed.

\section{Statistical Analysis}

Statistical analysis was performed using SAS 6.12 (SAS Institute Inc., Cary, NC, USA). Mean values and standard deviations were calculated. Comparisons between different time points were made using Wilcoxon signed rank test. Values of $\mathrm{p}<0.05$ were considered statistically significant.

\section{RESULTS}

Most fractures occurred in the thoracolumbar junction. Anatomic distribution of the collapsed vertebrae was as follows: T12 ( $n=5)$, L1 $(n=5), L 2(n=1)$, and L3 $(n=1)$. Mean age of the patients was 71.3 years (range, 61-80 years). As previously mentioned above, all patients had a severely osteoporotic spine with a mean $\mathrm{T}$-score of -4.02 (Table 1 ).

\section{Restoration of Vertebral Height}

Mean preoperative vertebral body height loss was 55.4\%.

Table 1. Clinical and radiological data for the patients

\begin{tabular}{|c|c|c|c|c|c|c|c|c|c|c|c|c|}
\hline \multirow{2}{*}{ Case } & \multirow{2}{*}{$\begin{array}{l}\text { Age/ } \\
\text { sex }\end{array}$} & \multirow{2}{*}{ Level } & \multicolumn{3}{|c|}{ Height loss (\%) } & \multicolumn{3}{|c|}{ Cobb angle $\left({ }^{\circ}\right)$} & \multirow{2}{*}{$\mathrm{BMD}$} & \multirow{2}{*}{$\begin{array}{c}\text { Cleft } \\
\text { component }\end{array}$} & \multirow{2}{*}{ Complication } & \multirow{2}{*}{ Result } \\
\hline & & & Preop & 7 Days & 6 Months & Preop & 7 Days & 6 Months & & & & \\
\hline 1 & $69 / \mathrm{F}$ & T12 & 70 & 50 & 55 & 25 & 10 & 12 & -3.4 & Gas & - & Excellent \\
\hline 2 & $72 / \mathrm{F}$ & L1 & 80 & 24 & 35 & 22 & 5 & 24 & -4.1 & Gas & T12 adjacent fracture & Poor \\
\hline 3 & $74 / \mathrm{F}$ & T12 & 45 & 25 & 40 & 18 & 18 & 38 & -3.5 & Fluid & T11 adjacent fracture & Poor \\
\hline 4 & $61 / \mathrm{F}$ & L1 & 40 & 25 & 30 & 25 & 8 & 15 & -4.7 & Gas & - & Excellent \\
\hline 5 & $67 / \mathrm{F}$ & L1 & 45 & 30 & 35 & 24 & 8 & 15 & -4.5 & Gas & Asymptomatic cement leakage & Good \\
\hline 6 & $72 / \mathrm{M}$ & L1 & 70 & 35 & 40 & 25 & 10 & 17 & -4.2 & Gas & - & Good \\
\hline 7 & $64 / \mathrm{F}$ & T12 & 35 & 20 & 20 & 20 & 10 & 12 & -3.6 & Fluid & - & Good \\
\hline 8 & $72 / \mathrm{F}$ & T12 & 30 & 20 & 25 & 25 & 10 & 15 & -3.9 & Fluid & - & Good \\
\hline 9 & $80 / \mathrm{M}$ & $\mathrm{L} 2$ & 65 & 45 & 45 & 20 & 10 & 15 & -4.5 & Gas & - & Excellent \\
\hline 10 & $72 / \mathrm{F}$ & L3 & 80 & 50 & 60 & - & - & - & -4.2 & Fluid & - & Fine \\
\hline 11 & $74 / \mathrm{F}$ & T12 & 50 & 30 & 40 & 22 & 12 & 20 & -3.9 & Gas & T11 adjacent fracture & Fine \\
\hline 12 & $78 / \mathrm{F}$ & L1 & 55 & 25 & 40 & 21 & 10 & 10 & -3.7 & Gas & - & Good \\
\hline
\end{tabular}

Preop, preoperative; BMD, bone mineral densitometry. 
Simple lateral radiographs taken 7 days after the surgical procedure showed that vertebral height loss was $31.6 \%$. Despite the significant improvement compared to preoperative value, average vertebral height loss was $38.8 \%$ at 6 months after the procedure $(\mathrm{p}=0.020)$. During the follow-up period, vertebral height loss increased by 7.2 (Fig. 2).

\section{Overall Reduction of Kyphotic Deformity}

The kyphotic angle improved significantly from $22.5^{\circ} \pm 4.5^{\circ}$ (before surgery) to $10.1^{\circ} \pm 5.0^{\circ}$ ( 7 days after procedure). However, the improved alignments were not maintained as observed on a true lateral X-ray taken during the final follow-up evaluation. Six months after surgery, mean correction loss for the kyphotic deformity was a $7.4^{\circ}$ from the angle at 7 days after procedure (Fig. 3). At 6 months after procedure, there was no significant improvement compared to preoperative value $(\mathrm{p}=$ $0.068)$.

\section{Clinical Outcome}

Six months after balloon kyphoplasty, outcomes for the 12 patients were graded according to modified MacNab criteria. The outcomes for 3 patients were excellent, 2 patients had good results, another 2 had fine results, and the remaining 5 patients had poor outcomes. Prior to kyphoplasty, the mean pain score (from the visual analogue scale) was 8.0. This score decreased to 2.5 at 7 days after the procedure, indicating that pain had decreased. Despite the significant improvement compared to preoperative value, the mean pain score increased to 4.0 at 6 months after the procedure (Fig. 4).

Asymptomatic cement leakage into the paravertebral area was observed in 1 patient. However, no major complications such as spinal cord compression, pulmonary embolism, or serious infection were observed in any study participant. During the 6-month follow-up period, 3 patients sustained subsequent fractures in the adjacent upper level at 27, 42, and 62 days after the procedure. Vertebroplasty or kyphoplasty with bone cement augmentation was planned to treat these fractures. But they refused bone cement augmentation procedures. No remote fractures occurred in this study population (Figs. 5, 6).

\section{DISCUSSION}

Osteoporotic compression fractures are common and a leading cause of disability and morbidity in the elderly ${ }^{7}$. Kummell disease is known to be a rare complication of osteoporotic compression fractures. This condition is a manifestation of posttraumatic vertebral osteonecrosis that is initially asymptomatic and radiographically negative, but eventually presents as delayed vertebral collapse. Due to its rarity, few studies on this disease have been published and strict guideline of management do not exist. No standard management protocol or single definitive treatment is available for individuals with Kummell disease. Several factors such as age, bone quality, severity of pain, degree of kyphotic deformity, and neurological deficits should be considered when selecting treatment modalities.

It is thought that Kummell disease is progressive. Therefore, conservative treatment is not considered to be appropriate for most patients ${ }^{5,8)}$. Because most of these patients have severe osteoporosis or are of an advanced age, recent studies have focused on the effects of percutaneous vertebral augmentation

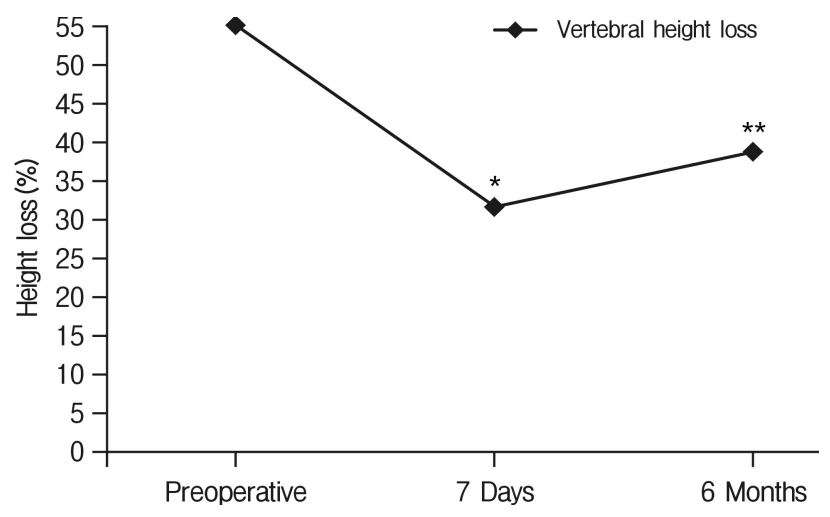

Fig. 2. Restoration of vertebral height. "Preoperative versus 7 days $(p=0.002)$. "Preoperative versus 6 moths $(p=0.002)$.

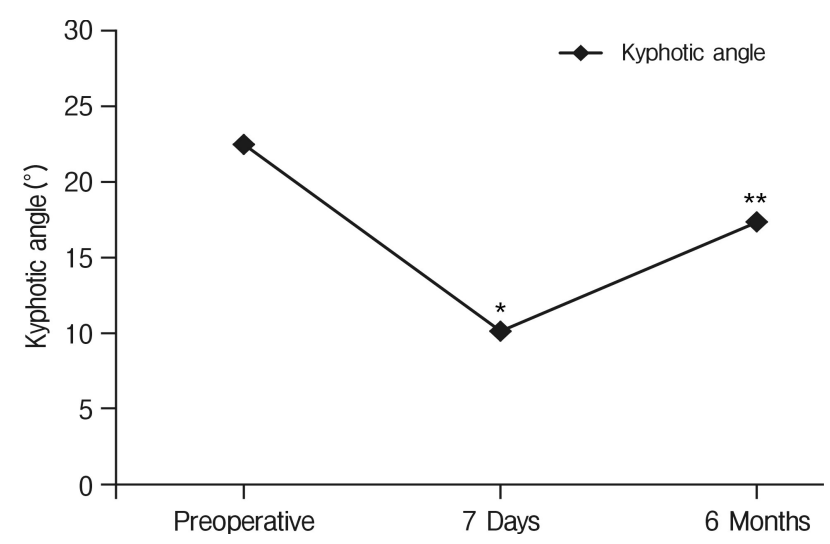

Fig. 3. Changes in kyphotic angle. "Preoperative versus 7 days $(p=0.005)$. Preoperative versus 6 months $(p=0.058)$.

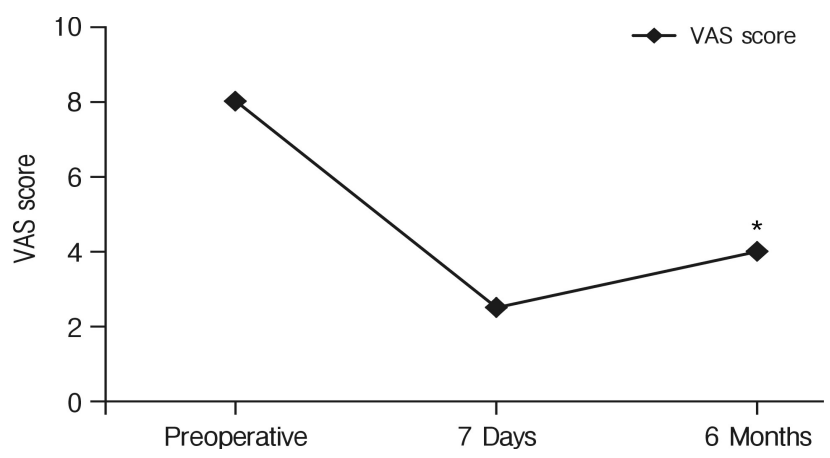

Fig. 4. The improvement of pain score. VAS, visual analogue scale. "Preoperative versus 6 months $(p<0.05)$. 

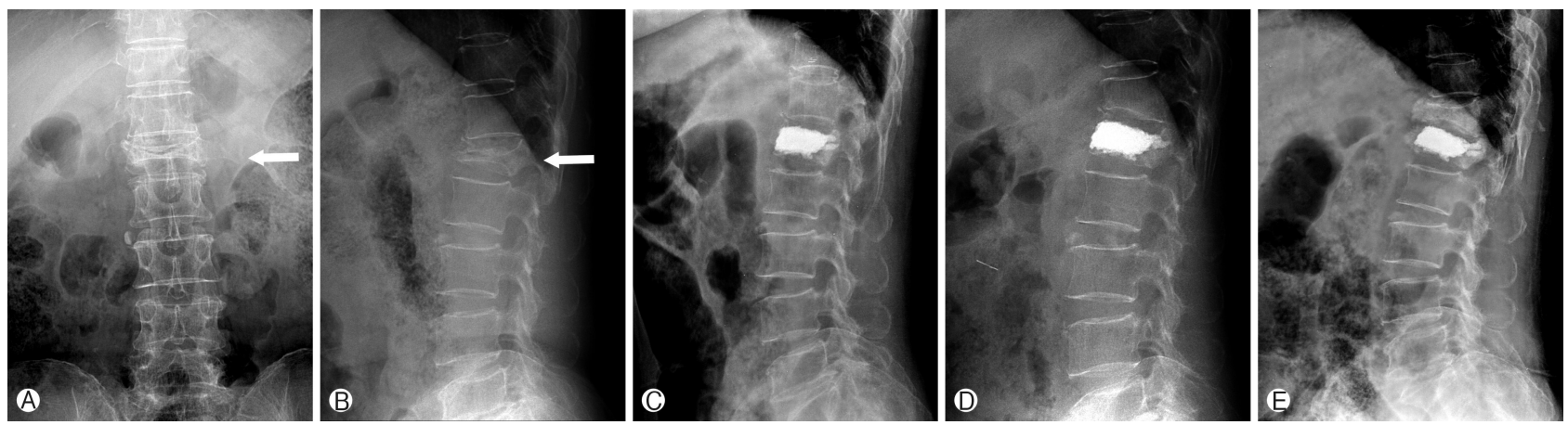

Fig. 5. Osteoporotic compression fractures with an intravertebral vacuum cleft at the L1 level in a 72-year-old female. (A, B) Preoperative simple radiographs show an intravertebral vacuum cleft indicative of Kummell disease (arrow). (C) Complete filling with bone cement without any leakage 7 days after balloon kyphoplasty. (D) Simple lateral radiograph taken 42 days after balloon kyphoplasty showing an adjacent vertebral fracture at the T12 level. (E) Simple lateral radiograph taken 6 months after the procedure reveals further collapse of the compression fracture.
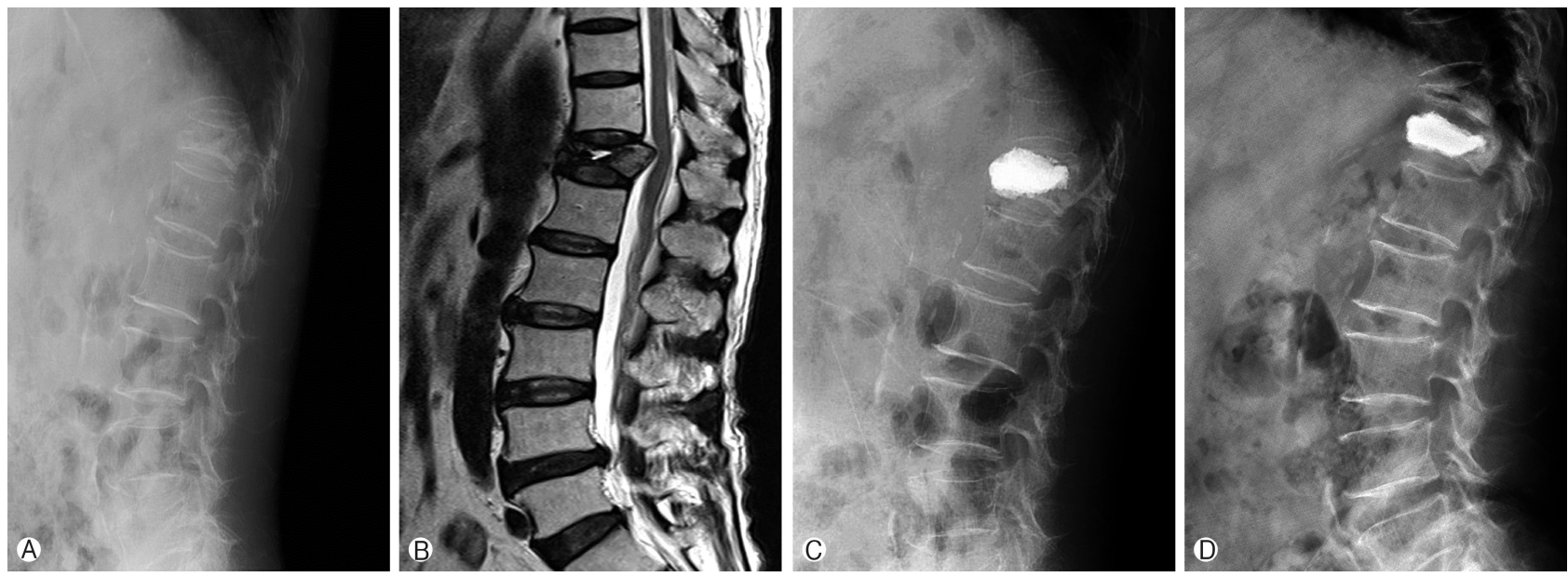

Fig. 6. Osteoporotic compression fracture with an intravertebral cleft at the T12 level in a 74-year-old female. (A, B) Simple lateral radiograph and T2-weighted magnetic resonance imaging show an osteoporotic compression fracture and intravertebral cleft. (C) Complete filling of the cleft with bone cement without any leakage is seen at 7 days after balloon kyphoplasty. (D) Simple lateral radiograph taken 62 days after balloon kyphoplasty reveals an adjacent vertebral fracture at the T11 level with increased kyphotic angle.

procedures such as vertebroplasty on pain relief, vertebral height restoration, and deformity correction. A retrospective study of 27 patients with IVC by Chen et al. ${ }^{2)}$ demonstrated that most patients were satisfied with the procedure outcomes in terms of pain relief. On the contrary, disappointing results have also been reported. Ha et al. ${ }^{4)}$ compared a group of 39 patients with or without an IVC. Twelve patients with an IVC reported a lesser degree of pain relief than the group without an IVC after vertebroplasty. Heo et al. ${ }^{5)}$ reported that the incidence of recollapse after percutaneous vertebroplasty is significantly higher in patients with osteonecrosis $(28.6 \%)$ than those without osteonecrosis (1.2\%). This group also suggested that vertebroplasty is not suitable for treating osteoporotic vertebral compression fractures with osteonecrosis or pseudoarthrosis. Furthermore, Lee et al. ${ }^{9)}$ showed that restored vertebral heights and corrected kyphotic angles were reaggravated, suggesting that vertebral osteonecrosis progresses after vertebroplasty or kyphoplasty have been performed.

In the present study, we performed balloon kyphoplasty to treat Kummell disease. The results of our study showed that balloon kyphoplasty had insufficient therapeutic outcomes in patients with Kummell disease like individuals with osteoporotic compression fractures without an IVC. Recently, Kim et $\mathrm{al}^{8}{ }^{8}$ reported that post-procedural kyphosis correction of more than $5^{\circ}$ with vertebroplasty could be a poor prognostic factor for patients with Kummell disease. They suggested that overcorrection of kyphosis would destabilize the fractured segment, resulting in further paravertebral soft tissue or ligament injury and persistent pain. Therefore, correction of the kyphotic angle (an advantage that balloon kyphoplasty has over vertebroplasty) seems to be unnecessary for treating Kummell disease. Moreover, PMMA injected into a fluid-filled cavity is believed to interdigitate less with the surrounding bone than that injected into partially intact trabecular bone. Therefore, PMMA can 
occupy cystic cavities without any mechanical interlock or biocompatibility, and is thus susceptible to dislodgment or fragmentation leading to a further kyphotic deformity ${ }^{10}$. For cases of Kummell disease in which the surrounding cortex has already been compromised, PMMA can easily leak out of the vertebral body and threaten the spinal canal ${ }^{11)}$. As a result, continuous careful follow-up observation after vertebroplasty or balloon kyphoplasty is recommended. In our study, 2 patients with poor functional outcomes sustained subsequent fractures in the adjacent upper vertebrae. No remote fractures were observed. This finding implies that the adjacent segment is the most vulnerable site to subsequent fractures after balloon kyphoplasty.

The IVC, which is visualized as linear transverse radiolucency on simple radiographs and characteristic of Kummell disease, is caused by the accumulation of gas in the center of a vertebral body or adjacent to an endplate. An IVC can also be found on CT images in which the distribution is more heterogeneous and irregular compared to that found on radiographs. Visualization of an IVC with MRI depends on whether the cleft is filled with gas or fluid. When filled with fluid during the early disease stage, the cleft produces a decreased signal on T1-weighted images and an increased signal on T2-weighted images. These features are highly suggestive of avascular necrosis. A peripheral zone of hyperintensity surrounding a hypointense band is occasionally seen on T2-weighted images (referred to as a 'double-line sign') and corresponds to an IVC. This sign may be indicative of sclerosis surrounding newly developed central granulation tissue formed during the later disease stages ${ }^{1)}$.

Based on our observations, we believe that the corrected kyphotic angles may be reaggravated with time after balloon kyphoplasty for Kummell disease. Due to the presence of a preexist ing cleft, it is not necessary to use balloons to create a cavity in patients with Kummell disease since a vacuum already exists. Strict postoperative spinal bracing and control of osteoporosis may be also helpful for preventing adjacent fractures and kyphotic deformity.

\section{CONCLUSION}

For the treatment of Kummell disease, the correction of kyphotic angle by balloon kyphoplasty seemed to be relatively ineffective. The development of subsequent fractures and aggravation of kyphotic deformities were the cause of poor prognosis after the procedure.

\section{CONFLICT OF INTEREST}

No potential conflict of interest relevant to this article was reported

\section{REFERENCES}

1. Baur A, Stäbler A, Arbogast S, Duerr HR, Bartl R, Reiser M: Acute osteoporotic and neoplastic vertebral compression fractures: fluid sign at MR imaging. Radiology 225:730-735, 2002

2. Chen LH, Lai PL, Chen WJ: Unipedicle percutaneous vertebroplasty for spinal intraosseous vacuum cleft. Clin Orthop Relat Res (435):148-153, 2005

3. Do HM, Jensen ME, Marx WF, Kallmes DF: Percutaneous vertebroplasty in vertebral osteonecrosis (Kummell's spondylitis). Neurosurg Focus 7:e2, 1999

4. Ha KY, Lee JS, Kim KW, Chon JS: Percutaneous vertebroplasty for vertebral compression fractures with and without intravertebral clefts. J Bone Joint Surg Br 88:629-633, 2006

5. Heo DH, Chin DK, Yoon YS, Kuh SU: Recollapse of previous vertebral compression fracture after percutaneous vertebroplasty. Osteoporos Int 20:473-480, 2009

6. Johnell O: Advances in osteoporosis: better identification of risk factors can reduce morbidity and mortality. J Intern Med 239: 299-304, 1996

7. Kim HS, Kim SH, Ju CI, Kim SW, Lee SM, Shin H: The role of bone cement augmentation in the treatment of chronic symptomatic osteoporotic compression fracture. J Korean Neurosurg Soc 48:490-495, 2010

8. Kim YJ, Lee JW, Kim KJ, Chung SK, Kim HJ, Park JM, et al: Percutaneous vertebroplasty for intravertebral cleft: analysis of therapeutic effects and outcome predictors. Skeletal Radiol 39: 757-766, 2010

9. Lee K, Lee SG, Kim WK, Yoo CJ, Park CW: Comparison vertebroplasty with kyphoplasty in delayed post-traumatic osteonecrosis of a vertebral body (Kummell's disease). Korean J Spine 5:70-76, 2008

10. Wang HS, Kim HS, Ju CI, Kim SW: Delayed bone cement displacement following balloon kyphoplasty. J Korean Neurosurg Soc 43:212-214, 2008

11. Yang SC, Chen WJ, Yu SW, Tu YK, Kao YH, Chung KC: Revision strategies for complications and failure of vertebroplasties. Eur Spine J 17:982-988, 2008

12. Young WF, Brown D, Kendler A, Clements D: Delayed posttraumatic osteonecrosis of a vertebral body (Kummell's disease). Acta Orthop Belg 68:13-19, 2002 